\title{
Delivery of antimicrobials to infected tissue macrophages
}

\author{
Irma A.J.M. Bakker-Woudenberg* \\ Department of Clinical Microbiology, Erasmus University Rotterdam, P.O. Box 1738, 3000 DR Rotterdam, The Netherlands
}

Received 24 March 1995; accepted 14 April 1995

Keywords: Liposomal antibiotic; Infection

\section{Contents}

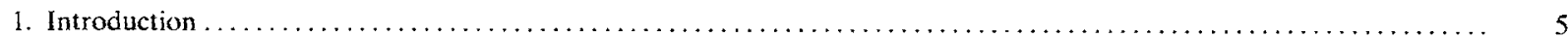

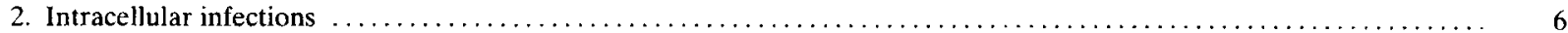

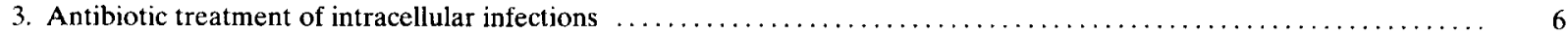

4. Liposomal encapsulation of antibiotic for treatment of intracellular infections $\ldots \ldots \ldots \ldots \ldots \ldots \ldots \ldots \ldots \ldots \ldots \ldots$

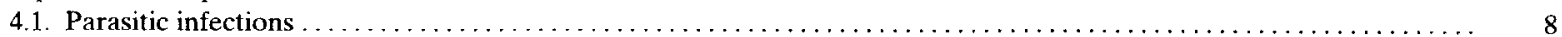

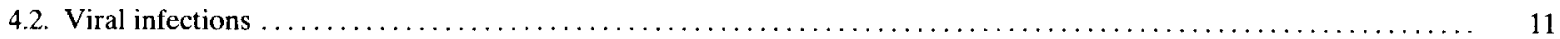

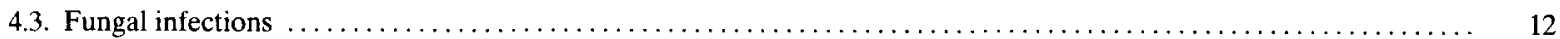

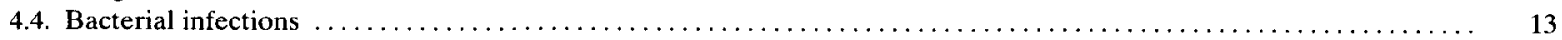

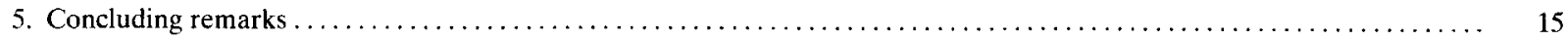

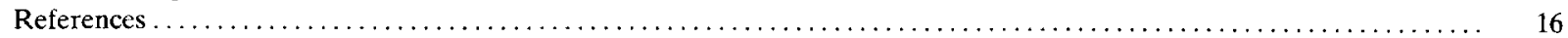

\section{Introduction}

The earliest therapeutic application of liposomal antimicrobial therapy was for the treatment of leishmaniasis in experimental animals [1,2], and published in 1978. In this case delivery of antimicrobial agent to infected Kupffer cells was accomplished using liposomes as carrier systems. Leishmania species are intracellular protozoa which are usually located in reticuloendothelial cells.

In a variety of intracellular parasitic, bacterial and viral infections the effect of liposomal encapsulation of antimicrobial agents on their therapeutic activity was further investigated. Based

* Corresponding author. Fax: +31 104364730. on the data obtained in experimental infections clinical studies in patients were started.

In the experimental models and clinical studies published to date 'classical' liposomes composed primarily of natural phospholipids and cholesterol were used. These liposomes, when administered intravenously, are unable to leave the general circulation and rapidly accumulate in cells of the mononuclear phagocyte system (MPS), particularly in liver and spleen. Accordingly, it is not surprising that most experimental data on the successful application of liposomes as carriers of antibiotics are derived from infections in MPS tissues caused by facultative or obligate intracellular pathogens.

The applicability of classical liposomes for delivery of antibiotic to extracellular infections in 
non-MPS tissues has been strongly limited by their short stay in blood due to the preferential uptake by the cells of the MPS. The use of liposomes as antibiotic carriers for treatment of infections outside the MPS would require a prolonged circulation half-life of liposomes. Recent progress in liposome design has yielded a new generation of liposomes characterized by long circulation half-lives and reduced hepatosplenic uptake. These liposome formulations, that show great promise for enhanced delivery of antibiotics in infectious diseases not restricted to MPS tissues, will not be discussed here.

\section{Intracellular infections}

Microorganisms that can survive and multiply intracellularly are protected from host defence mechanisms. Their intracellular location serves as a reservoir which is thought to be of importance in recurrent infections.

When invading pathogens have crossed one of the epithelial surfaces, they may spread through the body in various ways. The blood is the most effective vehicle of all for the spread of microorganisms. Macrophages are present in all major compartments of the body, and those lining the sinusoids in the liver, spleen, bone marrow and adrenals are particularly involved in removing foreign particles and microorganisms from the blood. These macrophages constitute the MPS, and the liver macrophages (Kupffer cells) are quantitatively the most important part of the MPS. After being removed from the blood, the behaviour of the microorganism in the macrophage becomes of great importance. Killing of the microorganism could mean termination of the infection, whereas microbial persistence and growth in the macrophage could lead to infection in the organ harbouring the macrophages, and hence to recurrent infection of the blood. Foci of infection in the liver, spleen and sometimes the bone marrow are characteristics of infections caused by intracellular pathogens.

Intracellular microorganisms can survive and even multiply intracellularly in various cell types, most of them even in macrophages and other phagocytes [3-5]. Obligate intracellular micro- organisms such as Mycobacterium leprae, and non-bacterial pathogens such as Chlamydia spp. or Leishmania spp. are host cell-dependent, cannot live in the extracellular space, and are exclusively found in the intracellular environment. In contrast, facultative intracellular microorganisms reside intracellularly transiently. Mycobacterium tuberculosis, Brucella spp., Salmonella spp., Legionella pneumophila, Listeria monocytogenes, and Neisseria gonorrhoeae are among facultative intracellular pathogens. These microorganisms are resistant to the intracellular killing mechanisms of these cells, and most of them actually multiply intracellulary. Normally phagocytosis of microorganisms is followed by fusion of the phagosome containing the bacteria with lysosomal granules present in the cell cytoplasm. Phagosome-lysosome fusion results in oxygen-dependent and oxygen-independent bacterial killing mechanisms in which the enzymes and cationic peptides present in the granules play an important role.

Different pathogens use various ways of evading intracellular killing to survive intracellularly: by escaping the phagosome followed by entry into the cytoplasm, or by inhibiting fusion of the lysosomes with the phagosomes, or by resisting the oxidative and non-oxidative killing mechanisms in phagolysosome.

\section{Antibiotic treatment of intracellular infections}

Eradication of intracellular infections is dependent on antibiotics, and is difficult due to the intracellular location of the microorganisms. In addition, the quiescent character of many intracellular pathogens prevents many antibiotics from expressing their activity.

Insight in the intracellular activity of antibiotics is of great importance. For successful treatment the penetrating capacity of the antibiotic into the infected cell as well as the subcellular distribution of antibiotics within the cell are important determinants [5-7].

The antibiotics enter cells via various mechanisms: passive transport via diffusion or active transport via transport proteins. Antibiotics dis- 
play great variations in their ability to penetrate into the host cell. Some antibiotics show poor or slow intracellular penetration; others achieve roughly similar intracellular and extracellular concentrations. In contrast, there are antibiotics that actually accumulate within the cell. However, the achievement of substantial intracellular antibiotic concentrations does not necessarily guarantee intracellular antimicrobial activity, even against susceptible infectious organisms. Sufficient antibiotic concentration in the intracellular location of the pathogen has to be achieved. The subcellular distribution of antibiotics is not uniform, showing important variations according to the antibiotic considered. The location of the intracellular pathogen is not uniform either.

The subcellular distribution of antibiotics is reviewed by Tulkens [6]. Intracellularly the antibiotic may be present in an cndosome, or in lysosomes, or may be located in the cytoplasm. The antibiotic activity is diversely influenced by various factors such as the local $\mathrm{pH}$, presence of proteins, enzymatic inactivation, ionic concentrations and other factors.

Aminoglycosides are too polar to pass across membranes, and are therefore only slowly taken up probably by endocytosis. As a result these agents are almost exclusively localized in lysosomes, and as a consequence exposed to acid $\mathrm{pH}$; they are not homogeneously distributed within cells. Only after long-term incubation relatively high concentrations are achieved in lysosomes. Penicillins and cephalosporins diffuse slowly through membranes of cells resulting in low intracellular concentrations. Even after longterm exposure of cells the intracellular concentrations are lower than the extracellular concentrations. These agents are found free in the cell cytosol. Lincosaminides can be found both in the cytosol and the lysosomes. Clindamycin accumulates in phagocytes, whereas lincomycin is not accumulated by phagocytes. Macrolides also show both a cytosolic and a lysosomal localization. As with lincosaminides differences are observed among the various derivatives. Most agents, among which erythromycin, show a marked intracellular accumulation. Fluoroquinolones also accumulate rapidly in cells, and are primarily found in the cytosol. Tetracyclines and chloramphenicol have been reported to accumulate in cells to a moderate extent. Rifampicin also accumulates in cells, and shows a high efficacy against sensitive bacteria in many studies. However, the accumulation ratios are relatively low, in spite of the high liposolubility of the agent. Rifampicin is distributed in the cytosol.

The intracellular antimicrobial activity of the antibiotic is also dependent of the microorganism's specific intracellular environment. Pathogens frequently reside within phagosomes. When phagosome-lysosome fusion occurs, the pathogen-containing vacuole is flooded with acidic and toxic lysosomal products. However, some bacteria escape from the phagosome to the cytoplasm or inhibit phagosome-lysosome fusion (as described earlier in this review).

It is clear that to predict intracellular activity of antibiotics is very complicated. In order to circumvent these difficulties investigators have proposed a more pragmatic approach. The antimicrobial activity of antibiotics can be evaluated by adding the compounds to pre-infected cells (phagocytes). After incubation the remaining intracellular microorganisms are counted. The studies available on the antimicrobial activity of a number of antibiotics against microorganisms ingested by mononuclear phagocytes are reviewed $[8,9]$, and show many apparently conflicting results. Various types of macrophages are used for these studies: animal macrophages which are usually collected from the peritoneal cavity of mice, rabbits or guinea pigs. The macrophages can be resident or exudate (the latter being cells harvested after the administration of an inflammatory stimulus). Peritoneal macrophages cannot be considered representative of all types of macrophage, as macrophages from various sites differ in a number of characteristics. It is difficult to obtain human tissue macrophages. Human monocytes isolated from the blood can also be used for such studies. The question is to what extent these mononuclear phagocytes are representative of human tissue macrophages in vivo.

In addition to the variation in cell types used for these studies, the duration of incubation and the type of pathogen (including its subcellular 
location) are all variables which must be carefully taken into account. Moreover there are methodological problems, especially when dealing with microorganisms that can grow both extracellularly and intracellularly. Caution must be exercised in drawing conclusions from the experimental data concerning the treatment of intracellular infections.

\section{Liposomal encapsulation of antibiotic for treatment of intracellular infections}

Improvement of the activity of antibiotics against microorganisms located inside cells has been achieved by entrapment of the antibiotic within liposomes. Classical liposomes, intravenously injected, are rapidly removed by the phagocytic cells of the liver and spleen and are at least partly localized in lysosomes where they are slowly degraded. Advantage is taken of this behaviour to treat infections located within these cells. The actual amount of classical liposomes taken up by the liver and spleen is dependent on the dose of lipid administered. With small doses most liposomes are retained by the liver. With large doses the clearance capacity of the liver may be overwhelmed, and uptake of liposomes by the spleen and bone marrow increases. Studies were performed in vitro in which various types of phagocytic or non-phagocytic cells infected with a variety of microorganisms were exposed to antimicrobial agents in the free versus liposome-entrapped form. In addition, in experimental models of intracellular infection the effect of liposomal encapsulation of antimicrobial agents on their therapeutic activity was investigated. In spite of the numerous in vitro and in vivo studies the clinical application of liposome-encapsulated antibiotics in the treatment of infections caused by intracellular pathogens in still limited.

In a variety of animal models of intracellular parasitic, bacterial and viral infections the effect of liposomal encapsulation of antimicrobial agents on their therapeutic activity was investigated. In general in all these studies an improved therapeutic effect and/or reduced toxicity resulting from encapsulation of the antibiotics in liposomes have been demonstrated. Most antibiotics are relatively ineffective for intracellular infections due to poor penetration into the cells or decreased activity intracellularly. Administration of antibiotic encapsulated in classical liposomes results in relatively high and prolonged concentrations of antibiotic in MPS tissues.

The rationale of this approach was based on the hypothesis that the liposomes are taken up by the cells containing the intracellular pathogens, and hence increase the ability of the antibiotic to reach its target, and to act in the corresponding environment. Surprisingly, only very few studies deal with the intracellular location of the liposomes and infectious organisms.

\subsection{Parasitic infections}

The first studies were published in 1978, and applied to intracellular parasitic infections caused by Leishmania spp. [1,2,10]. Diseases caused by the intracellular parasite Leishmania, a haemoflagellate protozoan, is a very important disease with a high prevalence in tropical and subtropical areas throughout the world. The parasite, which resides exclusively in macrophages in the MPS, has three different clinical manifestations: visceral (kala azar), mucocutaneous, and cutaneous leishmaniasis. In humans, the untreated visceral disease which affects liver, spleen and bone marrow, is the most severe and usually fatal, the other forms are severe (or fatal) and recurrent despite treatment with antimonial compounds. The antimonials are limited in effectiveness because of severe systemic toxicities, particularly to the heart, kidney and liver. Macrophages are the preferred habitat of Leishmania spp. which multiply in the phagolysosome where they are resistant to digestion. Leishmania donovani amastigotes are able to survive and metabolize in the acidic environment $(\mathrm{pH}$ 4-5) found in the phagolysosomes, and in addition are innately resistant to the lysosomal enzymes. Leishmania major survives by preventing the triggering of the respiratory burst.

By Alving et al. [1,10] leishmaniasis was induced in hamsters by intracardial injection of Leishmania donovani. This resulted in a fatal infection in which the organism settles in the 
cells of the MPS in the liver and spleen. Animals were treated intracardially with antimonial agents in the free or liposome-encapsulated form. Parameters for efficacy were effects on death rates or the total numbers of parasites per liver in surviving animals determined from impression smears. The studies reveal an increase in therapeutic efficacy of antimonial compound resulting from administration in the liposomeencapsulated form. The liposomal agent was at least 350 times as effective as the free agent in suppressing experimental leishmaniasis. Liposomes of various lipid compositions were used. The therapeutic efficacy was influenced by the lipid composition and the charge of the liposomes. The investigators found by empirical testing [1] that the lipid combinations that gave optimum therapeutic efficacy in leishmaniasis in hamsters are those that contain cholesterol and synthetic highly saturated phospholipids. Liposomes that are neutral or negatively charged are often more effective than positively charged ones.

The rationale of this approach was based on the hypothesis that the liposomes are rapidly taken up by the same type of cells in which Leishmania organisms reside, i.e., the phagocytic cells of the MPS, predominantly the Kupffer cells of the liver. Electron microscopic evidence was presented that supports this hypothesis. Electron micrographs of liver and spleen showed intracellular parasites adjacent to liposomes. The multilamellar structures of the liposomes were often not well preserved, but in some cases individual lamellae were sharply defined.

The therapeutic studies in the model of leishmaniasis in hamsters were extended to other classes of antimonial compounds, 8-aminoquinolines, administered by various routes [11]. Liposomes containing these agents were effective when given intracardially, intraperitoneally or intramuscularly, but not when given subcutaneously or orally. One of the most potent agents was up to 1800 times more effective than the agent alone. Empty liposomes lacked therapeutic efficacy.

Some years later similar observations have been made in dogs. Domestic dogs and wild canine species are the reservoirs for visceral leishmaniasis in the Americas, in the Middle East, and the Mediterranean basin [12]. In dogs intravenous Leishmania donovani infection resulted in visceral leishmaniasis of a focal character. Effective parasite suppression in the spleen obtained for intravenous treatment with the liposome-encapsulated agent was 700 times more efficacious than the unencapsulated drug.

In the model of leishmaniasis in hamsters the influence of parasite virulence on the effectiveness of liposomes in the treatment of visceral leishmaniasis was investigated [13]. The distinctive types of infection varied with respect to death rate and hepatic parasite counts. In every case, regardless of the type of infection the liposome-encapsulated agent was far superior to the non-encapsulated agent. However, even when the liposomal agent was employed, in the most virulent infections a fraction of animals appeared to be refractory to treatment.

At the same time of the first studies of Alving et al. in $1978 \mathrm{New}$ et al. [2], from another laboratory, reported the successful use of liposomes as carriers of antimonials for intravenous treatment of experimental visceral leishmaniasis caused by Leishmania donovani in mice.

Two years later these investigators published studies performed in a model of cutaneous leishmaniasis caused by Leishmania major injected subcutaneously in mice [14]. In this infection the parasite is located in macrophages in peripheral tissues rather than in the liver. Free or liposomeencapsulated antimonial agent was administered by different routes (intralesion or intravenously). The efficacy of the antileishmanial agent was enhanced by entrapping it inside liposomes; after the cutaneous lesion had begun to develop the intravenous route of administration of liposomal agent was the most effective.

The studies in experimental leishmaniasis described clearly show that liposomes may be useful as carriers of the therapeutic agents to treat infectious diseases involving the mononuclear phagocyte system. In case of extremely toxic antimonial agents encapsulation of the agent and reduction of the dose required for effective therapy should minimize systemic toxicity. Less than $0.15 \%$ of an ordinary therapeutic dose can be used for equivalent therapy. 
The investigators have clearly shown in electron microscopic studies in infected hamsters that antileishmanial agents inside liposomes have been brought into close contact with amastigotes in liver Kupffer cells [15]. The parasites, enclosed in so-called parasitophorous vacuoles, are able to survive after fusion with the lysosomes. Several hours after injection of liposomes, large numbers of vesicles are found in macrophages either in phagosomes or in lysosomes. The lysosomes containing liposomes commonly come into close apposition to leishmanial parasites. Instances of apparent fusion of the lysosomal membranes with parasitophorous vacuolar membranes are often observed. As a result the drug-laden liposomes are actually enclosed within the parasitophorous vacuole. Dramatic disintegration of Leishmania parasites in hamster Kupffer cells after treatment of the hamster with liposomal antimonial drug was shown, with remnants of liposomes observed. The high efficiency and precision of this process, involving liposomes as drug carriers and lysosomes as liposome carriers, probably accounts for the remarkable efficacy of liposome-encapsulated drugs in chemotherapy of leishmaniasis.

In further studies investigations in experimental leishmaniasis covered other compounds, such as amphotericin B, which exhibit antifungal activity. Antifungal agents were used based on possible biochemical similarities between fungi and protozoa.

Mice with intravenously induced leishmaniasis were treated intravenously with various antifungal agents [16]. All the agents tested displayed enhanced therapeutic activity when administered in the liposomal form. In the case of amphotericin B, liposomes composed of hydrogenated lecithins, or containing cholesterol or ergosterol in the membrane, were least toxic to the host and had the highest therapeutic capability. Another study of visceral leishmaniasis in mice confirmed the observation that amphotericin B in the liposomal form is significantly more active than the free form [17].

Other investigators reported the treatment of models of cutaneous leishmaniasis caused by footpad inoculation with Leishmania tropica in two strains of mice [18].
In mice which develop only local infection intravenous liposomal amphotericin B did not result in a decreased number of tissue parasites. In the other mouse strain the foodpad infection resulted in dissemination of the organism and eventually death of mice; in this model there was a slight effect of liposomal amphotericin B equal to non-encapsulated amphotericin B.

Also hamsters infected intracardially and squirrel monkeys infected intravenously with Leishmania donovani, resulting in visceral leishmaniasis, were treated with liposomal amphotericin B by the same route of administration as the Leishmania inoculation [19]. The amphotericin B liposomes consisted of dimyristoyl phosphatidylglycerol-dimyristoylphosphatidylcholine (molar ratio, 3:7), the same liposomes as used by Lopez-Berestein et al. in human trials. Comparcd to non-encapsulated antimonial agents liposomal amphotericin B was far superior in both animal models.

Industrially prepared Amphocil ${ }^{(B)}$ (amphotericin B Colloidal Dispersion) was also applied by intracardial injection in hamsters with visceral leishmaniasis caused by intracardiac inoculation of Leishmania donovani [20]. Amphocil ${ }^{(B)}$ was 15 times (lightly infected animals) or 4 times (heavily infected animals) as active as non-encapsulated amphotericin B.

The antileishmanial activity of industrially prepared AmBisome ${ }^{\circledR}$ was investigated in vitro as well as in animals [21]. Mouse peritoneal macrophages infected with Leishmania donovani were exposed to antifungal agent. Both AmBisome ${ }^{(B)}$ and non-encapsulated amphotericin B effectively killed the intracellular amastigotes at equivalent concentrations. In mice intravenously infected with Leishmania donovani AmBisome $\left.{ }^{(}\right)$was more effective compared to non-encapsulated amphotericin $B$.

Recently it was reported that neoglycoprotein conjugation of liposomes increased their potency as macrophage drug delivery system in Leishmania donovani infection [22]. In vitro in infected murine peritoneal macrophages hamycin entrapped in conjugated liposomes eliminated intracellular amastigotes 1.5 times and 10 times more efficiently than did the agent when entrapped in non-conjugated liposomes and in the 
free form, respectively. In mice with visceral leishmaniasis hamycin in conjugated liposomes could completely eliminate splenic parasites, whereas when administered in non-conjugated liposomes it was only partly effective.

The first clinical application of liposome-encapsulated amphotericin B in leishmaniasis was reported in 1991 [23]. Successful treatment of a patient with Mediterranean multiply drug-resistant visceral leishmaniasis with AmBisome ${ }^{(B)}$ was achieved. When reviewed 2 months after treatment the patient was symptom-free and all laboratory tests were normal.

There is a growing number of recent case reports demonstrating the efficacy of AmBisome $^{(\circledR)}$ in the treatment of drug-resistant visceral leishmaniasis [24]. In addition, AmBisome ${ }^{(i)}$ appeared to be effective in oral leishmanial lesions [25] as well as antimony-resistant cutaneous leishmaniasis [26]. In endemic areas, visceral leishmaniasis is considered a HIVassociated opportunistic infection. AmBisome ${ }^{\circledR}$ was also used for treatment of visceral leishmaniasis in AIDS patients unresponsive to antimonial compounds, and appeared to be effective for treatment and prophylaxis [27-29].

In spite of these observations on successful treatment it is important to be aware that relapse may occur. In two reports AIDS patients with visceral leishmaniasis are described who responded to treatment with AmBisome ${ }^{\circledR}$; however infection recurred despite the administration of maintenance therapy before the second relapse $[30,31]$. The authors presume that patients coinfected with HIV and Leishmania have a large parasite burden; in such cases, amastigotes infect cells other than macrophages (e.g., epithelial cells and neutrophils), which might be an explanation for treatment failure.

Summarizing, the experimental and clinical studies suggest that the use of AmBisome ${ }^{(B)}$ in the treatment of visceral leishmaniasis warrants further investigation. In immunocompromised patients comparative studies of induction and maintenance therapy are urgently needed. Particularly in these patients visceral leishmaniasis has emerged as an important opportunistic infection, and is more prevalent; treatment is particularly problematic [32].

\subsection{Viral infections}

The natural targeting of liposomes to cells of the MPS was also exploited to investigate whether selective delivery of antiviral agents could be beneficial for the treatment of viral diseases. Regarding viral infections these are always intracellular infections, in which many types of cells are involved. Virus replication in macrophages can be an integral factor in the pathogenesis of certain systemic virus infections. Macrophages, particularly the Kupffer cells, are extremely important as a barrier to prevent access of virus to other cell types. Incapacity of Kupffer cells in this respect, for example in the case of Rift Valley fever virus, enables the virus to pass straight to the endothelial cells and to infect the hepatic cells. It should be possible to direct antiviral agents to viruses replicating within macrophages. Besides, liposomes might also be targeted to other cell types by incorporating the appropriate antibodies into them. In that respect they should be ideal carriers of drugs to intracellular organisms such as viruses.

Regarding viruses replicating inside macrophages, experimental studies were performed with Rift Valley fever virus, which replication initially occurs inside the Kupffer cells, and later in macrophages of other visceral organs. Administration of liposome-encapsulated ribavirin to mice led to ribavirin concentrations in the liver, the primary site of Rift Valley fever virus proliferation, that were fivefold greater than those attained with the same doses of free ribavirin [33]. Liposomal ribavirin protected mice against a rapidly lethal challenge with Rift Valley fever virus, whereas similar doses of free drug or empty liposomes had no detectable benefit.

Also in vitro studies were performed in cultured human monocyte-macrophages infected with the human immunodeficiency virus type-1 (HIV-1). Cells of the monocyte-macrophage lineage are important hosts for the HIV-1, and play a key role in the dissemination of the virus and pathogenesis of AIDS, providing long-term reservoirs for the virus. The infected cells were exposed to various antiviral agents in the free or liposome-encapsulated form [34]. The liposomal agent and the free agent were equally potent. 
The observations on cellular uptake suggest that the antiviral drug was taken up by the cells inside the vesicles, probably by endocytosis. It was also shown that the tissue distribution and plasma pharmacokinetics of antiviral agent were modified when administered in the liposome-encapsulated form [35]. Further studies are warranted to explore the use of liposomes in vivo in the chemotherapy of infections with HIV.

\subsection{Fungal infections}

Regarding fungal infections most studies are performed with the antifungal agent amphotericin B encapsulated in liposomes or bound to other lipid carriers with the main purpose of reduction of toxicity of this agent. Extensive animal studies have been performed with various preparations of liposomal or lipid-complexed amphotericin B [36-51]. Various animal models of fungal infections were used. Fungal pathogens mostly used were Candida spp., Cryptococcus neoformans, Histoplasma capsulatum, Aspergillus spp., Coccidioides immitis and Blastomyces dermatitidis. Studies were performed in immunocompetent as well as immunodeficient animals. Various parameters for efficacy were used, such as animal survival rate, quantitative fungal cultures of infected organs as well as histological examination.

From clinical practice it is known that disseminated fungal infections require antifungal therapy; however, relapse after therapy is a common occurrence. Whereas it is known that certain intracellular fungal pathogens such as Cryptococcus neoformans and Histoplasma capsulatum particularly reside in the cells of the MPS, it is generally believed that relapse of infection after treatment of severe fungal infection may be caused by intracellularly surviving fungal pathogens. Fungi can be considered as facultative intracellular pathogens, as they are able to survive inside unactivated macrophages, and to grow out of these cells. Their intracellular location is thought to be of importance in recurrent infections. In our laboratory it was observed (data not yet published) that liposomal amphotericin B (AmBisome ${ }^{\circledR}$ ) is avidly taken up by macrophages in vitro, both by uninfected macrophages and macrophages infected with $C$. albicans. Uptake and degradation of AmBisome ${ }^{\circledR}$ in uninfected macrophages had no effects on subsequent $C$. albicans infection. Low intracellular antifungal activity was found for AmBisome ${ }^{(B)}$ compared to free amphotericin B, which is probably the result of slow intracellular degradation of AmBisome ${ }^{(\mathbb{R})}$ which is a solid liposome type.

The experimental studies on therapeutic efficacy clearly show that the antifungal activity of amphotericin B, dependent on the liposome or the lipid complex, is lower or equal compared to the non-encapsulated agent. However, in severe infections non-encapsulated amphotericin B could not be given at therapeutic levels because of toxicity; in the liposomal or lipid-complexed form amphotericin B, with its reduced toxicity, could be administered at drug levels capable of giving a therapeutic response. In other words, due to the decrease of toxicity these amphotericin B formulations display a marked increased therapeutic index. With respect to the industrially produced preparations AmBisome ${ }^{(}$, ABLC (amphotericin B Lipid Complex) and Amphocil $^{\circledR}$ (amphotericin B Colloidal Dispersion) it is evident that these have quite different structural and pharmacokinetic characteristics [52]. These pharmacokinetics depend to a large extent on the composition and particle size of the liposomes or the lipid complexes. Lipid structures such a ABLC and Amphocil ${ }^{\circledR}$ are rapidly taken up by the MPS; as a result relatively high amphotericin B concentrations are obtained in the liver and spleen without signs of major amphotericin B toxicity in these organs.

The promising results of the animal studies along with technical advances in liposomal preparation techniques have led to clinical application. Extensive clinical studies with various lipid formulations of amphotericin $B$ have been in progress for several years [53]. The efficacy of liposomal amphotericin $\mathrm{B}$ in patients with hepatosplenic candidiasis, a very protracted and difficult to treat infection, was good, and in patients with other infections encouraging. Most published clinical data on efficacy are available for AmBisome ${ }^{(B)}$ showing encouraging results in a 
variety of fungal infections in immunocompromised patients [54-58].

\subsection{Bacterial infections}

With respect to intracellular bacterial infections in MPS tissues in a number of animal infection models antibiotics were applied in the free or liposome-encapsulated form in most cases administered intravenously. Representative agents of various classes of antibiotics (aminoglycosides, penicillins, cephalosporins and quinolones) were used.

Most studies with antimicrobials encapsulated in liposomes are performed in experimental infections caused by organisms of the genus mycobacteria [59]. Mycobacteria are intracellular organisms that invade and multiply particularly within phagocytic cells. Infections caused by Mycobacterium tuberculosis (tuberculosis) or Mycobacterium leprae (leprosy), or Mycobacterium avium disseminated infections in AIDS patients are extremely common around the world. Treatment of mycobacterium infections is complicated, as sufficient concentrations of antimicrobials are needed within the intracellular compartment where the mycobacteria are located. In addition, resistance to antibiotics among mycobacteria is growing. Mycobacterium tuberculosis infects primarily pulmonary macrophages, while Mycobacterium avium complex infects particularly reticuloendothelial macrophages.

The facultative intracellular bacterium Mycobacterium tuberculosis secretes molecules that prevent phagosome-lysosome fusion. The phagocytic vacuoles remain free from lysosomal enzymes, bacteria can survive and grow. The obligate intracellular bacterium Mycobacterium leprae is able to escape from the phagosome into the cytoplasm, where it is protected from lysosomal enzymes. Even when phagosome-lysosome fusion occurs Mycobacterium spp. are resistant to lysosomal enzymes present in the phagolysosome due to their waxy cell wall that is very hydrophobic.

In mice intravenous inoculation with Mycobacterium tuberculosis resulted in infection of the spleen and lungs [60]. Intravenous injection of liposome-entrapped streptomycin led to a significant decrease in the spleen mycobacterium number, but not in the lungs, as compared to free streptomycin. Comparative pharmacokinetic studies reveal that streptomycin concentrations in spleen and liver were higher when administered in the liposomal form, whereas streptomycin concentrations in lungs and kidneys were independent from the drug form (liposomal or free) of streptomycin [61].

In another study rifampicin and isoniazid entrapped in liposomes were used for the treatment of murine tuberculosis [62]. The administration of liposome-entrapped drugs resulted in a significant reduction in the severity of the disease compared to the non-encapsulated drugs in terms of mycobacterium numbers in the spleen, as well as inflammation in the lungs determined by histology. It remains unanswered whether increased intracellular concentrations of drugs were achieved after administration in the liposomal form; it is known that rifampicin and isoniazid both penetrate cells well.

Recently it was shown in a model of Mycobacterium tuberculosis infection in mice that the antitubercular activity of rifampicin was considerably increased when it was encapsulated in liposomes. A further increase in the activity was observed when the macrophage activator tetrapeptide tuftsin was grafted on the surface of the drug-loaded liposomes [63]. It was clearly demonstrated that liposome targeting to macrophages could considerably increase the antitubercular activity of the agent.

A number of experimental models of Mycobacterium avium complex infection are described and used for treatment studies [64-72]. Particularly the beige mouse model of the disease was used. In most studies mice were intravenously infected. In beige mice this results in a chronic disseminated infection over a period of various weeks in which liver, spleen, lungs, kidneys and lymph nodes are involved. A variety of antimycobacterial agents among which the aminoglycosides amikacin, gentamicin, streptomycin and kanamycin, rifampicin and capreomycin were administered intravenously. The studies clearly demonstrate that liposome-based delivery of the therapeutic agent enhances its 
antimycobacterial effect. In most studies it is observed that the superior therapeutic effect of liposome-encapsulated agent is only seen in the spleen, and not in the lungs [60,61,64,66,68,71]. However, in some studies the increased therapeutic effect of liposomal agent over non-encapsulated agent was also observed in the lungs $[65,64,70,72]$. The discrepancies between these studies may be explained by differences in the lipid composition, and the total lipid doses used in the studies. Particularly the lipid dose is important, as with larger doses the ability of the liver to take up the liposomes from the circulation may be overwhelmed, and increasing amounts of liposomes are taken up by the spleen and bone marrow.

Pharmacokinetic studies reveal that concentrations of the antimycobacterial agents following administration in the liposome-encapsulated form are increased. In addition, data from in vitro studies suggest that intracellular delivery plays a role in the treatment of infections by Mycobacterium spp. The actual intracellular location of Mycobacterium avium complex is unknown. However, it has been shown that in vitro liposomes enhance the activity of antibiotics against Mycobacterium avium complex in phagocytic cells. In most cases monocytes or macrophages obtained from experimental animals in culture were used [65,73-75,70]; human monocytes/macrophages were also used [76-79]. The cells were infected with Mycobacterium avium complex and exposed to antibiotics among which the aminoglycosides amikacin, streptomycin and kanamycin, ciprofloxacin, clofazimin, clarithromycin, ofloxacin, azithromycin, rifabutin and rifampicin in the liposome-encapsulated or free form. In general the studies show that Mycobacterium avium complex was effectively killed when the infected cells were exposed to the liposomal agent, whereas the same concentration of the non-encapsulated agent alone or in combination with placebo liposomes did not show an antimicrobial effect. Even for clarithromycin and ofloxacin, antimicrobials that achieve high concentrations within macrophages, liposomal encapsulation significantly enhanced the activities [78]. It was observed that exposure of the infected cells to liposomal antibiotic at $4^{\circ} \mathrm{C}$ did not result in an intracellular antimicrobial effect, suggesting that liposomes need to be endocytosed to deliver their antibiotic intracellularly.

Electron microscopy of Mycobacterium avium complex-infected macrophages revealed that the bacteria were localized in phagosomes [77]. Some bacteria in infected macrophages exposed to either free or liposome-encapsulated ciprofloxacin showed signs of degradation. It was not possible to discern differences between mycobacteria inside macrophages treated with free ciprofloxacin and those inside macrophages treated with liposomal ciprofloxacin. Treatment of macrophages with placebo liposomes did not appear to cause bacterial degradation.

The in vitro activities of free and liposomal antimycobacterial agents against extracellular Mycobacterium avium complex and Mycobacterium tuberculosis were compared [80]. The results show that the antimycobacterial activities of certain drugs were maintained after the drugs were incorporated into liposomes.

The efficacy of liposomal gentamicin for the treatment of Mycobacterium avium complex bacteremia in AIDS patients was investigated [81]. Following intravenous administration of liposomal gentamicin twice weekly for 4 weeks mycobacterium numbers in blood fell substantially.

In animal models of infection caused by facultative intracellular bacteria such as Salmonella spp. [82-87], Brucella spp. [88, 89] and Listeria monocytogenes $[90,86]$ comparative studies were performed on the therapeutic efficacy of antibiotics in the liposome-encapsulated or non-encapsulated form. Mice or guinea pigs were infected intravenously; the intracellular bacteria concentrated primarily in the liver and spleen. Salmonella spp. and Brucella spp. owe their intracellular survival to their glycolipid capsule which is resistant to the action of lysosomal components following phagolysosome fusion (the non-oxidative killing mechanisms). Other bacterial species among which Listeria monocytogenes, Staphylococcus aureus and Fscherichia coli stimulate a normal respiratory burst, but are resistant to the effects of the toxic oxygen radicals by production of catalase or superoxide dismutase, enzymes that destroy hydrogen peroxide or superoxide.

In the treatment of these intracellular infec- 
tions in MPS tissues superior efficacy of antibiotics when administered intravenously in the liposome-encapsulated form was observed. The parameter for efficacy was survival of animals or bacterial recovery from infected organs. The antibiotics that were applied were the aminoglycosides streptomycin, kanamycin and gentamicin, ciprofloxacin, cephalotin and ampicillin.

Tissue distribution studies reveal substantially increased and more prolonged antibiotic concentrations particularly in the liver and spléen, following administration in the liposomal form.

Supplementary to these efficacy data obtained in models of bacterial infection, in vitro data on the role of liposomes in targeting of antibiotic to bacterial infected phagocytic cells also show the superiority of liposome-encapsulated antibiotics.

Phagocytic cells were murine or guinea pig macrophages, canine monocytes, or bovine monocytes/macrophages. Monocytes or macrophages infected with Salmonella spp. [91], Brucella spp. [88,89,92], Listeria monocytogenes [93,94], Staphylococcus aureus [95-97] or Escherichia coli [98] were exposed to the aminoglycosides streptomycin, amikacin, gentamicin, tobramycin and kanamycin, ampicillin, cephalothin and chloramphenicol in the free or liposome-cncapsulated form.

Studies were also performed with human monocytes/macrophages infected with Legionella pneumophila [99]. The intracellular bacteria were effectively killed by liposomal antibiotics, at concentrations that in the free form did not show an antimicrobial effect. The studies performed with Listeria monocytogenes demonstrated that by varying the lipid composition of the liposomes the intracellular degradation of the liposomes could be influenced and thereby the rate at which liposome-encapsulated agents are released and become available to exert their therapeutic action [93].

\section{Concluding remarks}

In view of their high affinity for the cells of the MPS, classical liposomes may serve as carriers of an appropriate antibiotic to intracellular sites of infection, particularly the liver and spleen. This is clearly demonstrated in a number of animal models of infection caused by facultative or obligate intracellular pathogens. After intravenous inoculation the microorganisms were rapidly cleared from the circulation and concentrated primarily in the liver and spleen. Often logarithmic growth of microorganisms in these organs was shown to occur.

Most studies have shown a significant improvement of liposome-encapsulated antimicrobials over free agent in the survival time of infected animals or a significant reduction in the number of microorganisms in infected organs. These observations are in line with the results obtained from in vitro studies in which infected phagocytic cells were exposed to liposome-encapsulated versus non-encapsulated antibiotic. The data suggest that administration of antibiotic in the liposome-encapsulated form may compensate for the poor penetration and/or retention of antibiotic within the cell. However, for antimicrobials that can achieve high concentrations within macrophages, liposomal encapsulation also resulted in significantly increased activities of the agents.

The concentration of antibiotics at the site of infection using liposomes allows treatment with a lower dosage. This is an important observation also with respect to potentially toxic antibiotics such as the aminoglycosides which despite their toxicity still play an important antimicrobial role; the encapsulation into liposomes will change the pharmacokinetics of these agents resulting in reduction of nephrotoxicity [100].

To what extent intracellular concentration of antibiotic is also achieved using liposomes for treatment is difficult to investigate. Supporting evidence for this has only been obtained from electron microscopic studies in experimental leishmaniasis. In addition, numerous in vitro studies using infected phagocytic cells suggest that intracellular delivery of antibiotic by means of liposomes plays a role in the successful treatment of intracellular infections. Only very few studies deal with the intraccllular location of the liposomes and the infectious organisms. Theoretically, the requirements for effective treatment of intracellular infections with liposome-encapsulated antibiotics are that next to intrinsic activity of antibiotic against the pathogen, the antibiotic must be at least considerably effective at the 
relatively low $\mathrm{pH}$ range of the phagolysosomal environment as well as relatively resistant to degradation in this environment.

Although liposome-encapsulated antibiotics are shown to be effective, in most studies the intracellular infection was not completely eliminated. Intracellular survival and multiplication of facultative or obligate intracellular microorganisms play a significant role in the pathogenesis of the diseases these organisms cause. Still liposomal encapsulation of antibiotic provides a therapeutic benefit by reducing the infectious load at least in the liver and spleen.

Recent experience from clinical application of liposomal amphotericin B for leishmaniasis in AIDS patients is encouraging. Unfortunately the occurrence of relapse of infection is also reported. Optimization of liposome formulation as well as treatment regimens are needed to provide further therapeutic advantage of liposomal encapsulation of antibiotics in these infections.

\section{References}

[1] Alving, C.R., Steck, E.A., Chapman, W.L., et al. (1978) Therapy of leishmaniasis: superior efficacies of liposomc-encapsulated drugs. Proc. Natl. Acad. Sci. USA 75, 2959-2963.

[2] New, R.R.C.. Chance, M.L., Thomas, S.C. and Peters. W. (1978) Antileishmanial activity of antimonials entrapped in liposomes. Nature 272, 55-56.

[3] Mims, C.A. (1986) The encounter of the microbe with the phagocytic cell. In: C.A. Mims (Ed.). The Pathogenesis of Infectious Diseases, 3rd edn. Academic Press, London, pp. 63-91.

[4] Stewart, J. and Weir, D.M. (1992) Immunity in bacterial infections. In: D. Greenwood, R. Slack and J. Peutherer (Eds.), Medical Microbiology, 14th edn Churchill Livingstone. Edinburgh, pp. 195-200.

[5] Donowitz, G.R. (1994) Tissue-directed antibiotics and intracellular parasites: complex interaction of phagocytes, pathogens and drugs. Clin. Infect. Dis. 19. 926-930.

[6] Tulkens, P.M. (1991) Intracellular distribution and activity of antibiotics. Eur. J. Clin. Microbiol. Infect. Dis. 10, 100-106.

[7] Butts, J.D. (1994) Intracellular concentrations of antibacterial agents and related clinical implications. Clin. Pharmacokine1. 27, 63-84.

[8] Van den Broek, P.J. (1989) Antimicrobial drugs, microorganisms, and phagocytes. Rev. Infect. Dis. 11, 213 245 .
[9] Van den Broek, P.J. (1991) Activity of antibiotics against microorganisms ingested by mononuclear phagocytes. Eur. J. Clin. Microb. Infect. Dis. 10, 114 118.

[10] Alving, C.R., Steck, E.A., Hanson, W.L., Loizeaux, P.S., Chapman, W.I.. and Waits, V.B. (1978) Improved therapy of experimental leishmaniasis by use of a liposome-encapsulated antimonial drug. Life Sci. 22, 1021-1026.

[11] Alving, C.R., Steck, E.A., Chapman, W.L., Waits, V.B., Hendricks, L.D., Swartz, G.M. and Hanson, W.L. (1980) Liposomes in leishmaniasis: therapeutic effects of antimonial drugs, 8-aminoquinolines, and tetracycline. Life Sci. 26, 2231-2238.

[12] Chapman, W.L., Hanson, W.L., Alving, C.R. and Hendricks, L.D. (1984) Antileishmanial activity of liposome-encapsulated meglumine antimonate in the dog. Am. J. Vet. Res. 45, 1028-1030.

[13] Alving, C.R., Waits, V.B. and Hanson, W.L. (1984) Liposomes in leishmaniasis: effects of parasite virulence on treatment of experimental leishmaniasis in hamsters. Ann. Trop. Med. Parasitol. 78, 279-286.

[14] New, R.R.C. and Chance, M.L. (1980) Treatment of experimental cutaneous leishmaniasis by liposome-entrapped Pentostam. Acta Trop. 37, 253-256.

[15] Alving. C.R., Weldon, J.S., Munnell, J.F. and Hanson, W.L. (1984) Liposomes in leishmaniasis: the lysosome connection. In: G. Gregoriadis. G. Poste, J. Senior and A. Trouet (Eds.), Receptor-mediated Targeting of Drugs. Plenum Press, New York, pp. 317-331.

[16] New, R.R.C.. Chance, M.L. and Heath, S. (1981) Antileishmanial activity of amphotericin and other antifungal agents entrapped in liposomes. J. Antimicrob. Chemother. $8,371-381$.

[17] Ahmad, I., Agarwal, A., Pal, A., Guru, P.Y., Bachhawat, B.K. and Gupta, C.M. (1991) Tissue distribution and antileishmanial activity of liposomised amphotericin B in Balb/c mice. J. Biosci. 14, 217-221.

[18] Panosian, C.B., Barza, M., Szoka, F. and Wyler, D.J. (1984) Treatment of experimental cutaneous leishmaniasis with liposome-intercalated amphotericin B. Antimicrob. Agents Chemother. 25, 655-656.

[19] Berman, J.D., Hanson, W.L., Chapman, W.L., Alving, C.R. and Lopez-Berestein, G. (1986) Antileishmanial activity of liposome-encapsulated amphotericin B in hamsters and monkeys. Antimicrob. Agents Chemother. 30, 847-851.

[20] Berman, J.D., Ksionski, G., Chapman, W.L., Waits, V.B. and Hanson. W.L. (1992) Activity of amphotericin B cholesterol dispersion (Amphocil) in experimental visceral leishmaniasis. Antimicrob. Agents Chemother. 36 , 1978-1980.

[21] Croft, S.L., Davidson, R.N. and Thornton, E.A. (1991) Liposomal amphotericin $\mathrm{B}$ in the treatment of visceral leishmaniasis. J. Antimicrob. Chemother. 28 (Suppl. B), $111-118$

[22] Kole, L., Sarkar, K., Mahato, S.B. and Das, P.K. (1994) Neoglycoprotein conjugated liposomes as macrophage 
specific drug carrier in the therapy of leishmaniasis. Biochem. Biophys. Res. Commun. 200, 351-358.

[23] Davidson, R.N., Croft, S.L., Scott, A., Maini, M., Moody, A.H. and Bryceson, A.D.M. (1991) Liposomal amphotericin $B$ in drug-resistant visceral leishmaniasis. Lancet 337, 1061-1062.

[24] Giacchino, R., Giambartolomei, G., Tasso, L., Timitilli, A., Castagnola, E., Brisigotti, M. and Micalizzi, C. (1993) Treatment with ljposomal amphotericin B of a child affected with drug-resistant visceral leishmaniasis. Trans. R. Soc. Trop. Med. Hyg. 87, 310.

[25] Baily, G.G., Pitt, M.A., Curry, A., Haboubi, N.Y, Tuffin, J.R. and Mandal, B.K. (1994) Leishmaniasis of the tongue treated with liposomal amphotericin B. J. Infect. 28, 327-331.

[26] Torre-Cisneros, J., Prada, J.L., Villanueva, J.L., Valverde, F. and Sánchez-Guijó, P. (1994) Successful treatment of antimony-resistant cutaneous leishmaniasis with liposomal amphotericin B. Clin. Infect. Dis. 18, 1024-1025.

[27] Lazanas, M.C., Tsekes, G.A., Papandreous, S., et al. (1993) Liposomal amphotericin B for leishmaniasis trcatmont of AIDS patients unresponsive to antimonium compounds. AIDS 7, 1018-1019.

[28] Torre-Cisneros, J., Villanueva, J.L., Kindelan, J.M., Jurado, K. and Sánchez-Guijó, P. (1993) Successtul treatment of antimony-resistant visceral leishmaniasis with liposomal amphotericin B in patients infected with human immunodeficiency virus. Clin. Infect. Dis. 17, 625-627.

[29] Dupla, M.L., Aguado, A.G., Uriol, P.L., Garcia, V.P., Ortega, E.V., Martinez, P.M. and Garcia-Puig, J. (1993) Efficacy of liposomal amphotericin B in the treatment and secondary prophylaxis of visceral leishmaniasis in HIV infected patients: report of two cases. J. Antimicrob. Chemother. 32, 657-659.

[30] McBride M., Linney, M., Claydon, E.J. and Weber, J. (1994) Visceral leishmaniasis following treatment with liposomal amphotericin B. Clin. Infect. Dis. 19, 362.

[31] Davidson, R.N. and Russo, R. (1994) Relapse of visceral leishmaniasis in patients who were coinfected with human immunodeficiency virus and who received treatment with liposomal amphotericin B. Clin. Infect. Dis. 19. 560 .

[32] Baily, G.G. and Nandy, A. (1994) Visceral leishmaniasis: more prevalent and more problematic. J. Infect. 29, 241-247.

[33] Kende, M., Alving, C.R., Rill, W.L., Swartz, G.M. and Canonico, P.G. (1985) Enhanced efficacy of liposomeencapsulated ribavirin against Rift Valley fever virus infection in mice. Antimicrob. Agents Chemother. 27, 903-907.

[34] Szebeni, J., Wahl, S.M., Betageri, G.V., et al. (1990) Inhibition of HIV-1 in monocyte/macrophage cultures by $2^{\prime}, 3^{\prime}$-dideoxycytidine-5'-triphosphate, free and in liposomes. AIDS Res. Hum. Retroviruses 6, 691-702.

[35] Désormeaux, A., Harvie, P., Perron, S., Makabi-Panzu, B., Beauchamp, D., Tremblay, M., Poulin, L. and Bergeron, M.G. (1994) Antiviral efficacy, intracellular uptake and pharmacokinetics of free and liposomeencapsulated 2',3'-dideoxyinosine. AIDS 8, 1545-1553.

[36] Patterson, T.F. and Andrioli, V.T. (1989) The role of liposomal amphotericin B in the treatment of systemic fungal infections. Eur. J. Cancer Clin. Oncol. 25 (Suppl. 2), $\$ 63-\$ 68$.

[37] Brajtburg, J., Powderly, W.G., Kobayashi, G.S. and Medoff, G. (1990) Amphotericin B delivery systems. Antimicrob. Agents Chemother. 34, 381-384.

[38] Gates, C. and Pinney, R.J. (1993) Amphotericin B and its delivery by liposomal and lipid formulations. J. Clin. Pharm. Ther. 18, 147-153.

[39] Lopez-Berestein, G.. Mehta, R., Hopfer, R.L., et al. (1983) Treatment and prophylaxis of disseminated infection due to Candida albicans in mice with liposome-encapsulated amphotericin B. J. Infect. Dis. 147, 939-945.

[40] Graybill, J.R., Craven, P.C., Taylor, R.L., Williams, D.M. and Magee, W.E. (1982) Treatment of murine cryptococcosis with liposome associated amphotericin B. J. Infect. Dis. 145, 748-752.

[41] Taylor, R.L., Williams, D.M., Craven, P.C., Graybill, J.R., Drutz, D.J. and Magee, W.E. (1982) Amphotericin B in liposomes: a novel therapy for histoplasmosis. Am. Rev. Respir. Dis. 125, 610-611.

[42] Lopez-Berestein, G., Hopfer, R.L., Mehta, R.. Mehta, K., Hersh, E.M. and Juliano, R.L. (1984) Liposomeencapsulated amphotericin B for treatment of disseminated Candidiasis in neutropenic mice. J. Infect. Dis. 150, 278-283.

[43] Clark, J.M., Whitney, R.R., Olsen. S.J., George, R.J., Swerdel, M.R., Kuuselman, L. and Bonner, D.P. (1992) Amphotericin B lipid complex therapy of experimental fungal infections in mice. Antimicrob. Agents Chemother. 35, 615-621.

[44] Clemons, K.V. and Stevens, D.A. (1992) Efficacies of amphotericin B lipid complex (ABLC) and conventional amphotericin $\mathrm{B}$ against murine coccidioidomycosis. J. Antimicrob. Chemother. 30, 353-363.

[45] Gondal, J.A., Swartz, R.P. and Rahman, A. (1989) Therapeutic evaluation of free and liposome-encapsulated amphotericin B in the treatment of systemic candidiasis in mice. Antimicrob. Agents Chemother. 33, 1544-1548.

[46] Adler-Moore, J.P., Chiang, S.M., Satorius, A., et al. (1991) Treatment of murine candidosis and cryptococcosis with unilamellar liposomal amphotericin B formulation (AmBisome). J. Antimicrob. Chemother. 28 (Suppl. B), 63-71,

[47] Patterson, T.F., Miniter, P., Dijkstra, J., Szoka, F.C., Ryan, J.L. and Andrioli, V.T. (1989) Treatment of experimental invasive Aspergillosis with novel amphotericin B/cholesterol-sulfate complexes. J. Infect. Dis. $159,717-724$.

[48] Clemons, K.V. and Stevens, D.A. (1991) Comparative efficacy of amphotericin B colloidal dispersion and amphotericin B deoxycholate suspension in treatment of murine coccidioidomycosis. Antimicrob. Agents Chemother. 35, 1829-1833. 
[49| I Iostetler, J.S., Clemons, K.V., Hanson, L.H. and Stevens, D.A. (1992) Efficacy and safety of amphotericin B colloidal dispersion compared with those of amphotericin B deoxycholate suspension for treatment of disseminated murine cryptococcosis. Antimicrob. Agents Chemother. 36, 2656-2660.

[50] Van Etten, E.W.M., Van den Heuvel-de Groot, C. and Bakker-Woudenberg, I.A.J.M. (1993) Efficacies of amphotericin B-desoxycholate (Fungizone), liposomal amphotericin B (AmBisome), and Fluconazole in the treatment of systemic candidosis in immunocompetent and leukopenic mice. J. Antimicrob. Chemother. 32. 723-739.

[51] Clemons, K.V. and Stevens, D.A. (1993) Therapeutic efficacy of liposomal formulation of amphotericin B (AmBisome) against murine blastomycosis. J. Antimicrob. Chemother. 32, 465-472.

[52] Janknegt, R., De Marie, S., Bakker-Woudenberg, I.A.J.M. and Crommelin, D.J.A. (1992) Liposomal and lipid formulations of amphotericin B. Clin. Pharmacokinet. 23, 279-291.

[53] De Marie S., Janknegt, R. and Bakker-Woudenberg. I.A.J.M. (1994) Clinical use of liposomal and lipidcomplexed amphotericin B. J. Antimicrob. Chemother. 33, $907-916$.

[54] Chopra, R., Blair, S., Strang, J., Cervi, P., Patterson, K.G. and Goldstone, A.H. (1991) Liposomal amphotericin B (AmBisome) in treatment of fungal infections in neutropenic patients. J. Antimicrob. Chemother. 28 (Suppl. B), 93-104.

[55] Ringdén, O., Meunier, F., Tollemar, J., et al. (1991) Efficacy of amphotericin B encapsulated in liposomes ( $\wedge \mathrm{mBisome}$ ) in the treatment of invasive fungal infections in immunocompromised patients. J. Antimicrob. Chemother. 28 (Suppl. B), 73-82.

[56] Meunier, F.. Prentice, H.G. and Ringdén, O. (1991) Liposomal amphotericin B (AmBisome): safety data from a phase II/III clinical trial. J. Antimicrob. Chemother. 28 (Suppl. B), 83-91.

[57] Chopra, R., Fielding. A. and Goldstone, A.H. (1992) Successful treatment of fungal infections in neutropenic patients with liposomal amphotericin B (AmBisome)A report on 40 cases from a single centre. Leukemia Lymphoma 7 (Suppl.), 73-77.

[58] Coker, R.J., Viviani, M., Gazzard, B.G., Du Pont. B., Pohle, M.D. and Murphy, S.M. (1993) Treatment of cryptococcosis with liposomal amphotericin B (AmBisome) in 23 patients with AIDS. AIDS 7, 829-835.

[59] Bermudez. L.E. (1994) Use of liposome preparation to treat mycobacterial infections. Immunobiology 191, $578-583$

[60] Vladimirsky, M.A. and Ladigina, G.A. (1982) Antibacterial activity of liposome-encapsulated streptomycin in mice infected with Mycobacterium tuberculosis. Biomedicine 36, 375-377.

[61] Ladigina, G.A. and Vladimirsky, M.A. (1986) The comparative pharmacokinetics of ${ }^{3} \mathrm{H}$-dihydrostreptomycin in solution and liposomal form in normal and
Mycobacterium tuberculosis infected mice. Biomed. Pharmacother. 40, 416-420.

[62] Orozco, L.C., Quintana, F.O., Beltrán, R.M., De Moreno, I., Wasserman, M. and Rodriguez, G. (1986) The use of rifampicin and isoniazid entrapped in liposomes for the treatment of murine tuberculosis. Tubercle 67, 91-97.

[63] Agarwal, A., Kandpal, H., Gupta, H.P., Singh, N.P. and Gupta. C.M. (1994) Tuftsin-bearing liposomes as rifampin vehicles in treatment of tuberculosis in mice. Antimicrob. Agents Chemother. 38, 588-593.

[64] Düzgüncs, N., Pcrumal, V.K., Kesavalu, L., Goldstein, J.A., Debs, R.J. and Gangadharam, P.R.J. (1988) Enhanced effect of liposome-encapsulated amikacin on Mycobacterium avium-M.intracellulare complex infection in beige mice. Antimicrob. Agents Chemother. 32 , 1404-1411.

[65] Saito, H. and Tomioka, H. (1989) Therapeutic efficacy of liposome-entrapped rifampin against Mycobacterium avium complex infection induced in mice. Antimicrob. Agents Chemother. 33, 429-433.

[66] Cynamon, M.H., Swenson, C.E., Palmer, G.S. and Ginsberg, R.S. (1989) Lipusume-encapsulatedamikacin therapy of Mycobacterium avium complex infection in beige mice. Antimicrob. Agents Chemother. 33, 1179-1183.

[67] Bermudez, L.E., Yau-Young, A.O., Lin, J.P., Cogger, J. and Young, L.S. (1990) Treatment of disseminated Mycobacterium avium complex infection of beige mice with liposome-encapsulated aminoglycosides. J. Infect. Dis. 161, 1262-1268.

[68] Klemens, S.P., Cynamon, M.H., Swenson, C.E. and Ginsberg, R.S. (1990) Liposome-encapsulated gentamicin therapy of Mycobacterium avium complex infection in beige mice. Antimicrob. Agents Chemother. 34, 967-970.

[69] Gangadharam, P.R.J., Ashtekar, D.A., Ghori, N., Goldstein, J.A., Debs, R.J. and Düzgünes, N. (1991) Chemotherapeutic potential of free and liposome-encapsulated streptomycin against experimental Mycobacterium avium complex infections in beige mice. J. Antimicrob. Chemother. 28, 425435.

[70] Tomioka, H., Saito, H., Sato, K. and Yoneyama, T. (1991) Therapeutic efficacy of liposome-encapsulated kanamycin against Mycobacterium intracellulare infection induced in mice. Am. Rev. Respir. Dis. 144, 575579.

[71) Düzgünes, N., Ashtekar, D.R., Flasher, D.L., et al. (1991) Treatment of Mycobacterium avium-intracellulare complex infection in beige mice with free and liposome-encapsulated streptomycin: role of liposome type and duration of treatment. J. Infect. Dis. 164, $143-151$.

[72] Le Conte, P., Le Gallou, F., Potel, G., Struillou, L., Baron, D. and Drugeon, H.B. (1994) Pharmacokinetics, toxicity and efficacy of liposomal capreomycin in disseminated Mycobacterium avium beige mouse model. Antimicrob. Agents Chemother. 38, 2695-2701.

[73] Jansons, V.K. and Soggin, A. (1990) Clofazimine and 
liposomes enhance the susceptibility of intracellular Mycobacterium avium towards rifabutin. Curr. Microbiol. 20, 261-264.

[74] Kesavalu, L., Goldstein, J.A., Debs, R.J., Düzgünes, N. and Gangadharam, P.R.J. (1990) Differential effects of free and liposome-encapsulated amikacin on the survival of Mycobacterium avium complex in mouse peritoneal macrophages. Tubercle 71, 215-218.

[75] Ashtekar, D., Düzgünes, N. and Gangadharam, P.R.J. (1991) Activity of free and liposome-encapsulated streptomycin against Mycobacterium avium complex (M^C) inside peritoneal macrophages. J. Antimicrob. Chemother. 28, 615-617.

[76] Bermudez, L.E.M., Wu, M. and Young, L.S. (1987) Intracellular killing of Mycobacterium avium complex by rifapentine and liposome-encapsulated amikacin. J. Infect. Dis. 156, 510-513.

[77] Majumdar, S., Flasher, D., Friend, D.S., et al. (1992) Efficacies of liposome-encapsulated streptomycin and ciprofloxacin against Mycobacterium avium- $M$. intracellulare complex infections in human peripheral blood monocyte/macrophages. Antimicrob. Agents Chemother. 36, 2808-2815.

[78] Onyeji, C.O., Nightingale, C.H., Nicolau, D.P. and Quintiliani, R. (1994) Efficacies of liposome-encapsulated clarithromycin and ofloxacin against Mycobacterium avium- $M$. intracellutare complex in human macrophages. Antimicrob. Agents Chemother. 38, 523527.

[79] Onyeji, C.O., Nightingale C.H., Nicolau, D.P. and Quintiliani, R. (1994) Activities of liposome-encapsulated azithromycin and rifabutin compared with that of clarithromycin against Mycobacterium avium-intracellulare complex in human macrophages. Int. J. Antimicrob. Agents 4, 281-289.

[80] Mehta, R.T., Keyhani, A., McQueen, T.J., Rosenbaum, B., Rolston, K.V. and Tarrand, J.J. (1993) In vitro activities of free and liposomal drugs against Mycobacterium avium- $M$. intracellulare complex and $M$. tuberculosis. Antimicrob. Agents Chemother. 37, 2584-2587.

[81] Nightingale, S.D., Saletan, S.L., Swenson, C.E., et al. (1993) Liposome-encapsulated gentamicin treatment of Mycobacterium avium-Mycobacterium intracellulare complex bacteremia in AIDS patients. Antimicrob. Agents Chemother. 37, 1869-1872.

[82] Desiderio, J.V. and Campbell, S.G. (1983) Liposomeencapsulated cephalothin in the treatment of experimental murine salmonellosis. J. Reticuloendoth. Soc. 34, 279-287.

[83] Tadakuma, T., Ikewaki, N., Yasude, T., Tsutsumi, M., Saito, S. and Saito, K. (1985) Treatment of experimental salmonellosis in mice with streptomycin entrapped in liposomes. Antimicrob. Agents Chemother. 28, 28 32.

[84] Swenson, C.E., Stewart, K.A., Hammett, J.L., Fitzsimmons, W.E. and Ginsberg, R.S. (1990) Pharmacokinetics and in vivo activity of liposome-encapsulated gentamicin. Antimicrob. Agents Chemother. 34, 235240 .
[85] Fierer, J., Hatlen, J., Liu, J.P., Estrella, D., Mihalko, P. and Yau-Young, A. (1990) Successful treatment using gentamicin liposomes of Salmonella dublin infections in mice. Antimicrob. Agents Chemother. 34, 343-348.

[86] Fattal, E., Rojas, J., Youssef, M., Couvreur, P. and Andremont, A. (1991) Liposome-entrapped ampicillin in the treatment of experimental murine listeriosis and salmonellosis. Antimicrob. Agents Chemother. 35, 770772.

[87] Magallanes, M., Dijkstra, J. and Fierer, J. (1993) Liposome-incorporated ciprofloxacin in treatment of murine salmoncllosis. Antimicrob. Agents Chemother. 37. 2293-2297.

[88] Dees. C., Fountain, M.W., Taylor, J.R. and Schultz, K.D. (1985) Enhanced intraphagocytic killing of Brucella abortus in bovine mononuclear cells by liposomes containing gentamicin. Vet. Immunol. Immunopathol. 8, 171-182.

[89] Fountain, M.W., Weiss, S.J., Fountain, A.G., Shen, A. and Lenk, R.P. (1985) Treatment of Brucella canis and Brucella abortus in vitro and in vivo by stable plurilamellar vesicle-encapsulated aminoglycosides. J. Infect. Dis. 152, 529-535.

[90] Bakker-Woudenberg, I.A.J.M., Lokerse, A.F., Roerdink, F.H., Regts, D. and Michel, M.F. (1985) Free versus liposome-entrapped ampicillin in treatment of infection due to Listeria monocytogenes in normal and athymic (nude) mice. J. Infect. Dis. 151, 917-924.

[91] Desiderio, J.V. and Campbell, S.G. (1983) Intraphagocytic killing of Salmonella typhimurium by liposome-encapsulated cephalothin. J. Infect. Dis. 148, $563-570$.

[92] Dees, C. and Schultz, R.D. (1990) The mechanism of enhanced intraphagocytic killing of bacteria by liposomes containing antibiotics. Vet. Immunol. Immunopathol. 24, 135-146.

[93] Bakker-Woudenberg, I.A.J.M., Lokerse, A.F. and Roerdink, F.H. (1989) Antibacterial activity of liposome-entrapped ampicillin in vitro and in vivo in relation to the lipid composition. J. Pharmacol. Exp. Ther. 251, 321-327.

[94] Forestier, F., Gerrier, P., Chaumard, C., Quero, A.M., Couvreur, P. and Labarre, C. (1992) Effect of nanoparticle-bound ampicillin on the survival of Listeria monocytogenes in mouse peritoneal macrophages. J. Antimicrob. Chemother. 30, 173-179.

[95] Bonventre, P.F. and Gregoriadis, G. (1978) Killing of intraphagocytic Staphylococcus aureus by dihydrostreptomycin entrapped within liposomes. Antimicrob. Agents Chemother. 13, 1049-1051.

[96] Fountain, M.W., Dees, C. and Schultz, R.D. (1981) Enhanced intracellular killing of Staphylococcus aureus by canine monocytes treated with liposomes containing amikacin, gentamicin, kanamycin and tobramycin. Curr. Microbiol. 6, 373-376.

[97] MacLeod, D.L. and Prescott, J.F. (1988) The use of liposomally-entrapped gentamicin in the treatment of bovine Staphylococcus aureus mastitis. Can. J. Vet. Res. $52,445-450$. 
[98] Stevenson, M., Baillie, A.J. and Richards, R.M.E. (1983) Enhanced activity of streptomycin and chloramphenicol against intracellular Escherichia coli in the J774 macrophage cell line mediated by liposome delivery. Antimicrob. Agents Chemother. 24, 742-749.

[99] Sunamoto, J., Goto, M., Iida. T.. Hara, K., Saito, A. and Tomonaga, A. (1985) Unexpected tissue distribution of liposomes coated with amylopectin derivatives and successful use in the treatment of experimental Legionnaires' disease. In: G. Gregoriadis, G. Poste, J. Senior and A. Trouet (Eds.), Receptor-mediated Targeting of Drugs. NATO ASI Series, Plenum Press, New York, pp. 359-371.

[100] Karlowsky, J.A. and Zhanel, G.G. (1992) Concepts on the use of liposomal antimicrobial agents: applications for aminoglycosides. Clin. Infect. Dis. 15, 654-667. 